\title{
A novel HDAC inhibitor OBP-801 and a PI3K inhibitor LY294002 synergistically induce apoptosis via the suppression of survivin and XIAP in renal cell carcinoma
}

\author{
TAKESHI YAMADA ${ }^{1,2}$, MANO HORINAKA ${ }^{1}$, MASAHIDE SHINNOH ${ }^{1,2}$, \\ TAKASHI YOSHIOKA ${ }^{1,3}$, TSUNEHARU MIKI ${ }^{2}$ and TOSHIYUKI SAKAI ${ }^{1}$ \\ Departments of ${ }^{1}$ Molecular-Targeting Cancer Prevention, ${ }^{2}$ Urology and ${ }^{3}$ Obstetrics and Gynecology, \\ Kyoto Prefectural University of Medicine, Kawaramachi-Hirokoji, Kamigyo-ku, Kyoto 602-8566, Japan
}

Received April 22, 2013; Accepted June 10, 2013

DOI: 10.3892/ijo.2013.2042

\begin{abstract}
Renal cell carcinoma (RCC) is resistant to traditional cancer therapies such as radiation therapy and chemotherapy. The use of targeted therapies has improved the clinical outcomes of patients with metastatic RCC. However, most patients acquire resistance against targeted therapies over time. We report that the combination of the novel histone deacetylase (HDAC) inhibitor OBP-801, also known as YM753 and the phosphatidylinositol 3-kinase (PI3K) inhibitor LY294002 synergistically inhibits cell growth and induces apoptosis in RCC cells. This combination activated caspase-3, -8 and -9 and the pan-caspase inhibitor zVAD-fmk significantly reduced the apoptotic response to the treatment with OBP-801 and LY294002. Moreover, the combined treatment induced intracellular reactive oxygen species (ROS) and the radical scavenger $N$-acetyl-L-cysteine (NAC) blocked the intracellular ROS and apoptosis induced by OBP-801 and LY294002. The co-treatment with OBP-801 and LY294002 markedly decreased survivin and the X-linked inhibitor of apoptosis protein (XIAP) protein levels, but Bcl-2 family members were not altered by the OBP-801/LY294002
\end{abstract}

Correspondence to: Professor Toshiyuki Sakai, Department of Molecular-Targeting Cancer Prevention, Graduate School of Medical Science, Kyoto Prefectural University of Medicine, KawaramachiHirokoji, Kamigyo-ku, Kyoto 602-8566, Japan

E-mail: tsakai@koto.kpu-m.ac.jp

Abbreviations: RCC, renal cell carcinoma; HDAC, histone deacetylase; PI3K, phosphatidylinositol 3-kinase; ROS, reactive oxygen species; NAC, $N$-acetyl-L-cysteine; XIAP, X-linked inhibitor of apoptosis protein; SAHA, suberoylanilide hydroxamic acid; mTOR, mammalian target of rapamycin; PIP3, phosphatidylinositol 3,4,5-trisphosphate; IAP, inhibitor of apoptosis; IRS-1, insulin receptor substrate-1; IGF-I, insulin-like growth factor-I; NF- $\kappa \mathrm{B}$, nuclear factor- $\kappa \mathrm{B}$

Key words: HDAC inhibitor, PI3K inhibitor, renal cell carcinoma, survivin, X-linked inhibitor of apoptosis protein co-treatment. These alterations were restored by NAC treatment. The transient transfection of survivin and XIAP reduced the apoptotic response to the OBP-801/LY294002 co-treatment. Additionally, OBP-801 was significantly more effective than SAHA, another HDAC inhibitor, in the combination with LY294002 against 786-O cells. Taken together, these results strongly suggest the combination of OBP-801 and LY294002 to be a promising treatment for RCC.

\section{Introduction}

Renal cell carcinoma ( $\mathrm{RCC}$ ), the most common malignant tumor of the kidney, accounts for $2-3 \%$ of adult malignancies. It causes about 102,000 deaths worldwide per year (1-3). Several molecular-targeting agents for RCC have been developed; the multi-tyrosine kinase inhibitors sorafenib and sunitinib, and the mammalian target of rapamycin (mTOR) inhibitors everolimus and temsirolimus. One of the major activities of these agents against RCC has been believed to be their angiogenesis-inhibitory effect. Despite the success of these agents, drug resistance is an urgent problem, which underscores the need for new treatment strategies to improve clinical outcomes (4-6).

Histone deacetylase (HDAC) inhibitors are promising anticancer agents that induce growth arrest, differentiation and apoptosis in various types of tumor cell lines $(7,8)$. We identified OBP-801, also known as YM753, as a novel HDAC inhibitor with attractive pharmacodynamic and pharmacokinetic properties by screening for a p21 ${ }^{\mathrm{WAF} 1 / \mathrm{Cip} 1}$-inducing agent (9). OBP-801 exerted the most potent HDAC-inhibitory activity tested; it was about 50 times more effective than SAHA, the most clinically used HDAC inhibitor (9).

Phosphatidylinositol 3-kinase (PI3K) is a major signaling component downstream of growth factor receptor tyrosine kinases (10). Phosphatidylinositol 3,4,5-trisphosphate (PIP3) generated by PI3K at the cell membrane is a lipid second messenger and contributes to the activation of the serinethreonine protein kinase Akt $(10,11)$. The PI3K-Akt signaling pathway is a key regulator of cell growth through many downstream targets. Therefore, the PI3K inhibitor LY294002 can inhibit cell growth and cause apoptosis also in RCC cells (11-15). 
Previous reports showed that co-treatment with an HDAC inhibitor and a PI3K inhibitor was effective against ovarian cancer, cervical cancer, non-small cell lung cancer, colon cancer, chronic myeloid leukemia and cutaneous T-cell lymphoma by downregulating XIAP and Mcl-1 (16-21). The PI3K-Akt pathway is well known to be upregulated in most RCC, but the combination of a PI3K inhibitor and an HDAC inhibitor has not been examined in RCC cells. Therefore, we examined if this combination was effective on RCC and found that the co-treatment of the PI3K inhibitor LY294002 with the novel HDAC inhibitor OBP-801 drastically induced apoptosis through the strong suppression of survivin as well as XIAP via ROS production. This is the first report that the downregulation of survivin at least partially contributes to the synergistic effect of the HDAC inhibitor with the PI3K inhibitor.

\section{Materials and methods}

Reagents. OBP-801 (Oncolys BioPharma, Tokyo, Japan), LY294002 (Cell Signaling Technology, Beverly, MA, USA), SAHA (Biomol Research Laboratories, Plymouth Meeting, PA, USA) and zVAD-fmk (R\&D Systems, Minneapolis, MN, USA) were dissolved in DMSO. $N$-acetyl-L-cysteine (NAC) was purchased from Nacalai Tesque (Kyoto, Japan).

Cell culture. Human renal cancer 786-O and ACHN cell lines were maintained in RPMI-1640 and DMEM, respectively. Culture media were supplemented with $10 \%$ FBS, glutamine (2 mM for RPMI-1640 and $4 \mathrm{mM}$ for DMEM), $100 \mathrm{U} / \mathrm{ml}$ penicillin and $100 \mu \mathrm{g} / \mathrm{ml}$ streptomycin. Cell cultures were incubated at $37^{\circ} \mathrm{C}$ in a humidified atmosphere of $5 \% \mathrm{CO}_{2}$.

Cell viability assay. The number of viable cells was determined using a Cell Counting kit-8 assay according to the manufacturer's instructions (Dojindo Laboratories, Kumamoto, Japan). After the incubation of cells for $72 \mathrm{~h}$ with the indicated concentrations of OBP-801 or LY294002, the kit reagent WST-8 was added to the medium and cells were incubated for a further $4 \mathrm{~h}$. The absorbance of samples $(450 \mathrm{~nm})$ was determined using a scanning multiwell spectrophotometer (DS Pharma Biomedical, Osaka, Japan).

Detection of apoptosis. DNA fragmentation was quantified by the percentage of hypodiploid DNA (sub-G1). Cells were harvested from culture dishes, washed with PBS and treated with PBS containing $0.1 \%$ Triton X-100. Cells were then treated with RNase A (Sigma, St. Louis, MO, USA) and the nuclei were stained with propidium iodide (Sigma). DNA content was measured using a FACSCalibur flow cytometer and CellQuest software (Becton-Dickinson, Franklin Lakes, NJ, USA). For each experiment, 10,000 events were analyzed.

Western blot analysis. Western blot analysis was carried out as described previously (22). The following antibodies were purchased from the indicated sources: rabbit polyclonal antibodies for anti-survivin (R\&D Systems), anti-caspase-3 (Cell Signaling Technology), anti-Bcl-2 (Abcam, Cambridge, UK), anti-BAX and anti-Bcl-xL (Santa Cruz Biotechnology, Santa Cruz, CA, USA) and mouse monoclonal antibodies for anti-XIAP (R\&D Systems), anti-caspase-8, -9 (MBL,
Nagoya, Japan) and anti- $\beta$-actin (Sigma) were used as primary antibodies. The signal was detected using a Chemilumi-one chemiluminescent kit (Nacalai Tesque).

Plasmid DNA transfection. The pCMV6-XL5 control, pCMV6-XL5/survivin and pCMV6-XL5/XIAP plasmid constructs were purchased from OriGene Technologies (Rockville, MD, USA). 786-O cells were seeded at $1 \times 10^{5}$ cells per well in 6-well plates without antibiotics. After $24 \mathrm{~h}$, plasmid DNA $(4 \mu \mathrm{g})$ was transfected into cells using HilyMax transfection reagent (Dojindo Laboratories) according to the manufacturer's instructions. Four hours after the transfection, the medium was replaced with fresh medium and cells were treated with or without OBP-801/LY294002 for $48 \mathrm{~h}$ and then harvested.

Measurement of intercellular ROS. For the measurement of ROS production, cells were treated with $10 \mu \mathrm{M} 5$-(and-6)-chloromethyl-2,7-dichlorodihydrofluorescein diacetate acetyl ester (CM-H ${ }_{2}$ DCFDA) (Molecular Probes, Carlsbad, CA, USA). After 30 min of incubation with $\mathrm{CM}-\mathrm{H}_{2} \mathrm{DCFDA}$, fluorescence was monitored in the FL-1 channel by FACSCalibur using the CellQuest software.

Combination index. We calculated the combination index for OBP-801 and LY294002 using CalcuSyn 2.0 software (Biosoft, Great Shelford, UK).

Statistical analysis. Data were expressed as mean \pm SD of three determinations. Statistical analysis was performed using the Student's t-test. Samples were considered significantly different at $\mathrm{P}<0.05$.

\section{Results}

The combination of OBP-801 and LY294002 synergistically inhibits cell growth and induces apoptosis in RCC 786-O cells. To examine the growth-inhibitory effect of OBP-801 or LY294002 alone, we assessed the viable cell number of 786-O cells after $72 \mathrm{~h}$ of treatment with the indicated concentrations of agents. Each agent was effective against cell growth in a dose-dependent manner in 786-O and ACHN cells (Fig. 1A). Interestingly, co-treatment with low-dose OBP-801 and LY294002 synergistically inhibited cell growth less than that of cells treated with either single agent in 786-O and ACHN cells (Fig. 1B). Moreover, the combination index (CI) values for OBP-801 and LY294002 were $<1.0$, indicating a synergistic effect for the inhibition of cell growth (Fig. 1C). To clarify the mechanisms of synergistic inhibitory effects on cell growth by the combination of OBP-801 and LY294002, we investigated the effects of the combination on apoptosis by measuring the sub-G1 population. OBP-801 or LY294002 alone only weakly induced apoptosis, but the co-treatment with OBP-801 and LY294002 more remarkably induced apoptosis in 786-O cells (Fig. 1D). These results indicate that the combination of OBP-801 and LY294002 synergistically inhibits cell growth and induces apoptosis in 786-O cells.

The combination of OBP-801 and LY294002 induces caspase-dependent apoptosis in 786-O cells. We investigated 
A
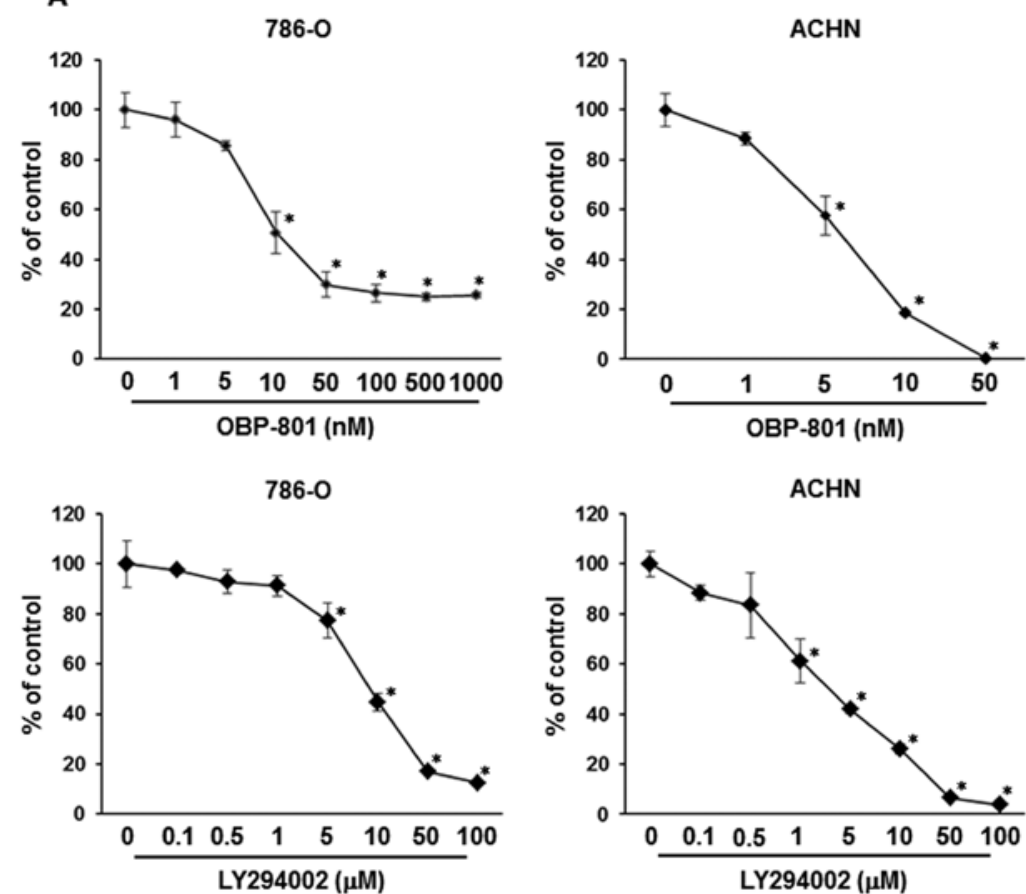

B

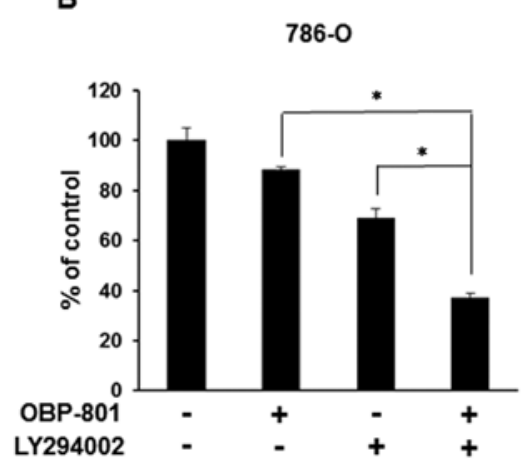

C

\begin{tabular}{|c|c|c|}
\hline $\begin{array}{c}\text { OBP-801 } \\
(\mathrm{nM})\end{array}$ & $\begin{array}{c}\text { LY294002 } \\
(\mu \mathrm{M})\end{array}$ & $\mathrm{Cl}$ \\
\hline 2 & 2.5 & 0.501 \\
\hline 4 & 5 & 0.376 \\
\hline 8 & 10 & 0.381 \\
\hline 16 & 20 & 0.712 \\
\hline
\end{tabular}

D

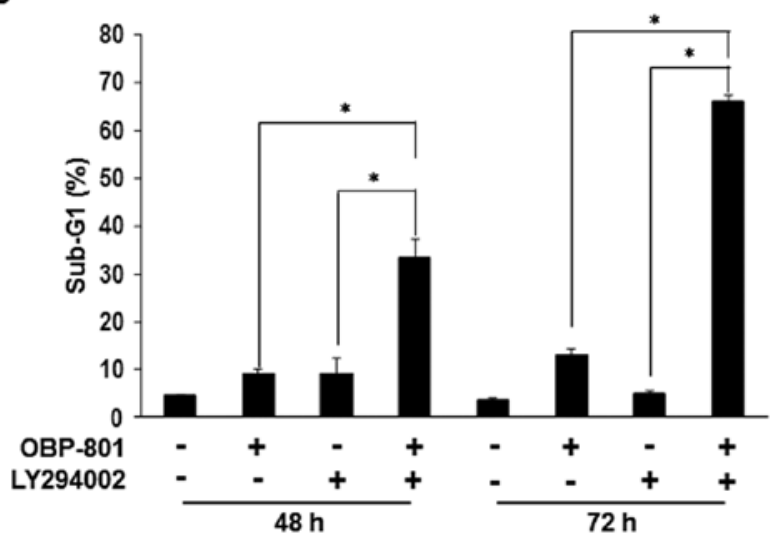

Figure 1. OBP-801 and LY294002 synergistically inhibit cell growth and induce apoptosis. (A) 786-O or ACHN cells were treated with DMSO alone (control) or the indicated concentrations of OBP-801 or LY294002. After incubation for $72 \mathrm{~h}$, viable cells were counted using Cell Counting kit-8. Columns, means of triplicate data; bars, $\mathrm{SD} ;{ }^{*} \mathrm{P}<0.05$, significantly different from that by DMSO. (B) 786-O or ACHN cells were treated with $4 \mathrm{nM}$ OBP-801 and/or $5 \mu \mathrm{M}$ LY294002. After incubation for $72 \mathrm{~h}$, viable cells were counted using Cell Counting kit-8. Columns, means of triplicate data; bars, $\mathrm{SD} ;{ }^{*} \mathrm{P}<0.05$, significantly different from that by either single agent. (C) The combination index (CI) of OBP-801 and LY294002 was calculated in 786-O cells. (D) 786-O cells were treated with $4 \mathrm{nM} \mathrm{OBP-} 801$ and/or $5 \mu \mathrm{M}$ LY294002 for 48 or $72 \mathrm{~h}$. Apoptosis (sub-G1) was determined using flow cytometry analysis. Columns, means of triplicate data; bars, SD; " $\mathrm{P}<0.05$.

whether the apoptosis induced by the combination of OBP-801 and LY294002 depends on caspases using the pan-caspase inhibitor zVAD-fmk. Treatment with zVAD-fmk effectively inhibited the apoptosis induced by the co-treatment with OBP-801 and LY294002 (Fig. 2A). Additionally, we performed western blotting of caspase-3, -8 and -9 . Treatment with OBP-801 or LY294002 alone did not induce the cleavage of caspases, but the co-treatment with OBP-801 and LY294002 induced caspase cleavage (Fig. 2B). These results suggest that the combination of OBP-801 and LY294002 induces apoptosis dependent on caspase in 786-O cells. 
A

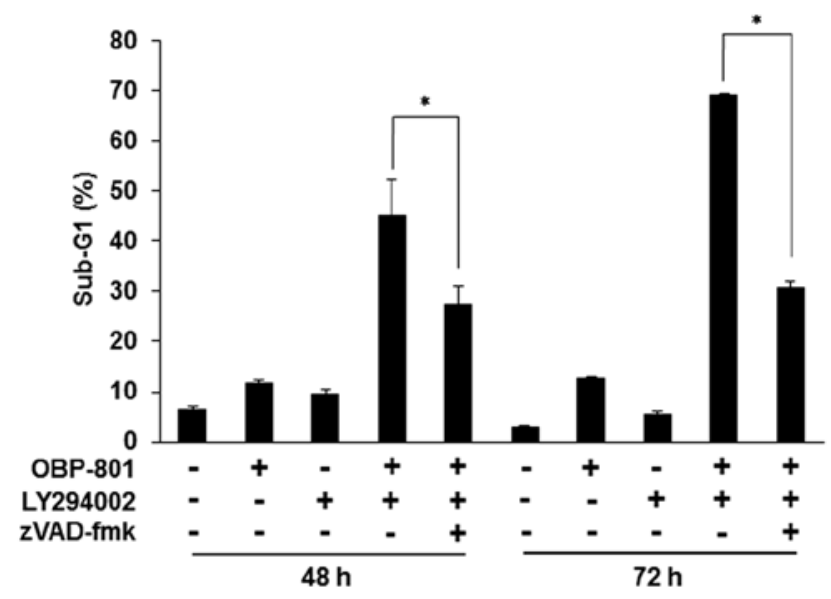

B

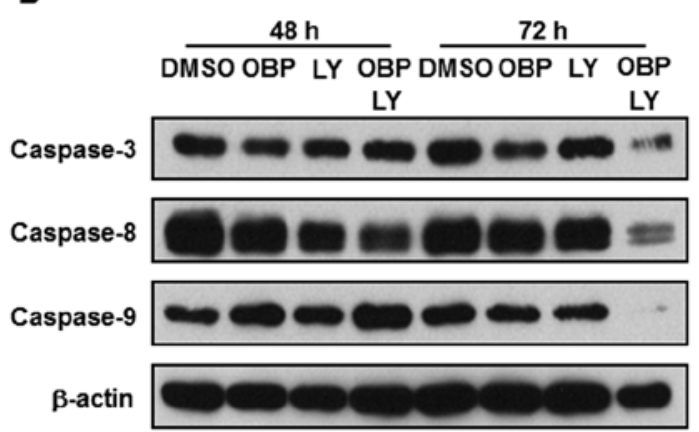

Figure 2. OBP-801 and LY294002 induce caspase-dependent apoptosis. (A) 786-O cells were treated with $4 \mathrm{nM}$ OBP-801 and/or $5 \mu \mathrm{M} \mathrm{LY} 294002$ with or without the pan-caspase inhibitor zVAD-fmk ( $20 \mu \mathrm{M})$ for 48 or $72 \mathrm{~h}$. The sub-G1 population was analyzed by flow cytometry. Columns, means of triplicate data; bars, SD; ${ }^{*} \mathrm{P}<0.05$. (B) Western blotting of caspase- $3,-8$ and $-9.786-\mathrm{O}$ cells were treated with $4 \mathrm{nM}$ OBP-801 and/or $5 \mu \mathrm{M} \mathrm{LY} 294002$ for 48 or $72 \mathrm{~h}$. $\beta$-actin is shown as a loading control.

ROS are responsible for the apoptosis induced by the combination of OBP-801 and LY294002 in 786-O cells. It has been reported that the apoptosis induced by the combination of a HDAC inhibitor and a PI3K inhibitor is associated with the intracellular accumulation of ROS (19). We also found that the co-treatment with OBP-801 and LY294002 induced intracellular ROS and the free radical scavenger, NAC, blocked the intracellular ROS induced by the co-treatment in 786-O cells (Fig. 3A). Moreover, NAC blocked OBP-801/ LY294002-induced apoptosis in 786-O cells (Fig. 3B). These results suggest that the apoptosis induced by the combination of OBP-801 and LY294002 is dependent on ROS production.

The combination of OBP-801 and LY294002 decreases protein levels of survivin and XIAP through ROS generation in 786-O cells. To clarify the molecular mechanism of the apoptosis induced by the combination of OBP-801 and LY294002, we performed western blot analysis. As shown in Fig. 4A, the expression of anti-apoptotic molecules such as survivin and XIAP with the co-treatment of OBP-801 and LY294002 was significantly lower than with either single agent. $\mathrm{Bcl}-2, \mathrm{BAX}$ and $\mathrm{Bcl}-\mathrm{xL}$ protein levels were not affected by the
A

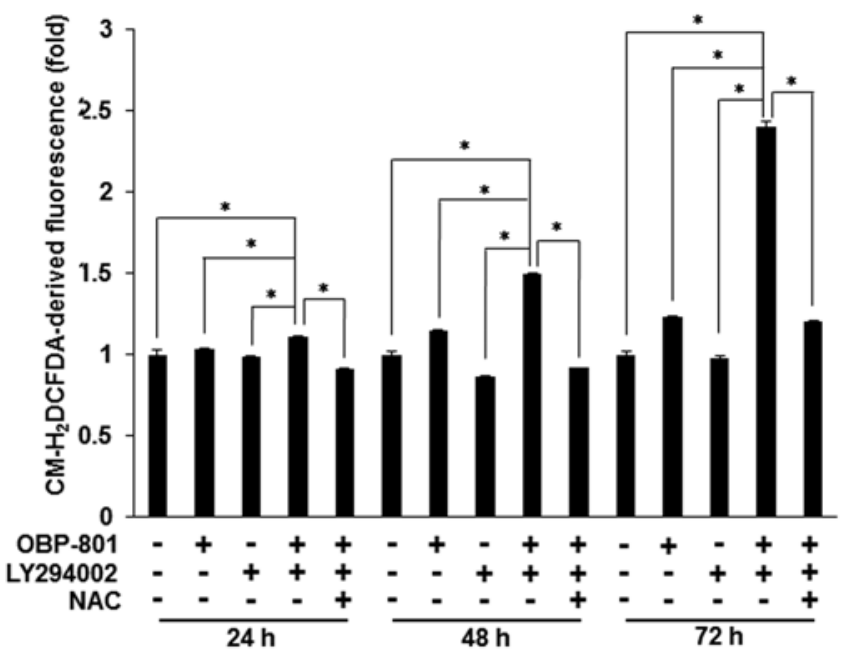

B

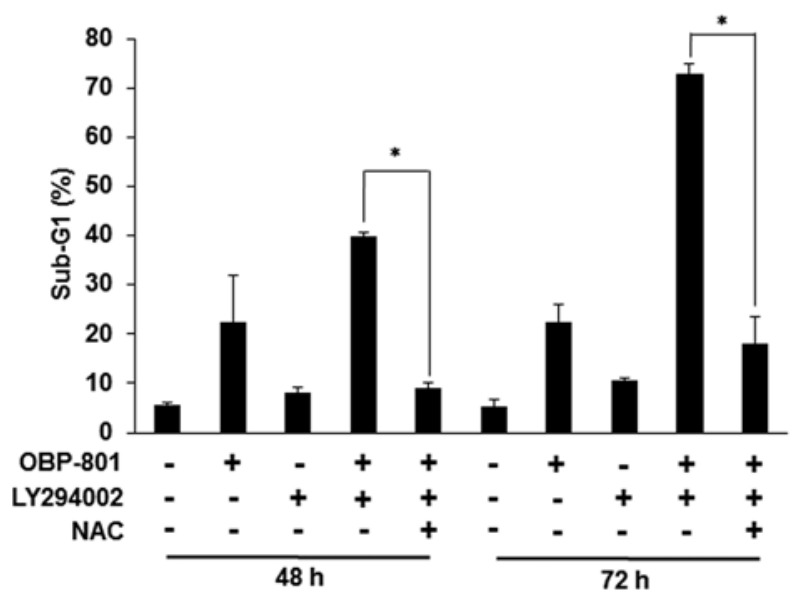

Figure 3. ROS are responsible for OBP-801/LY294002-induced apoptosis. (A) 786-O cells were treated with $4 \mathrm{nM} \mathrm{OBP-801}$ and/or $5 \mu \mathrm{M}$ LY294002, with or without NAC $(5 \mathrm{mM})$. After 24,48 or $72 \mathrm{~h}$ of treatment, ROS levels were analyzed using $\mathrm{CM}-\mathrm{H}_{2}$ DCFDA as described in Materials and methods. Columns, means of triplicate data; bars, SD; ${ }^{*} \mathrm{P}<0.05$. (B) 786-O cells were treated with $4 \mathrm{nM}$ OBP-801 and/or $5 \mu \mathrm{M} \mathrm{LY} 294002$, with or without NAC $(5 \mathrm{mM})$ for 48 or $72 \mathrm{~h}$. The sub-G1 population was analyzed by flow cytometry. Columns, means of triplicate data; bars, SD; "P<0.05.

co-treatment with OBP-801 and LY294002 (Fig. 4B). Next, we investigated further whether ROS generation could cause the downregulation of survivin and XIAP. As shown in Fig. 4C, the downregulation of survivin and XIAP was restored by NAC treatment. These results suggest that the downregulation of survivin and XIAP induced by the combination of OBP-801 and LY294002 is ROS-dependent.

Downregulation of survivin and XIAP is involved in the apoptosis induced by the combination of OBP-801 and LY294002. We examined whether overexpression of survivin and XIAP contributed to the resistance to the co-treatment with OBP-801 and LY294002. The effects of the overexpression of survivin and XIAP were confirmed by western blotting (Fig. 5A). As shown in Fig. 5B, the overexpression of survivin or XIAP partially suppressed OBP-801/LY294002-induced apoptosis, 
A

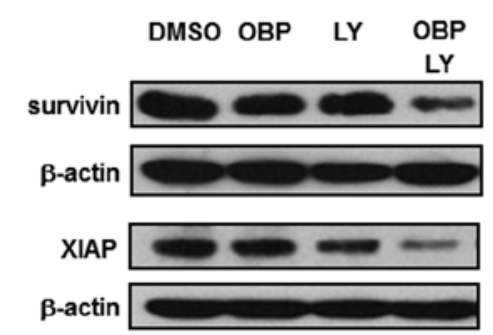

B

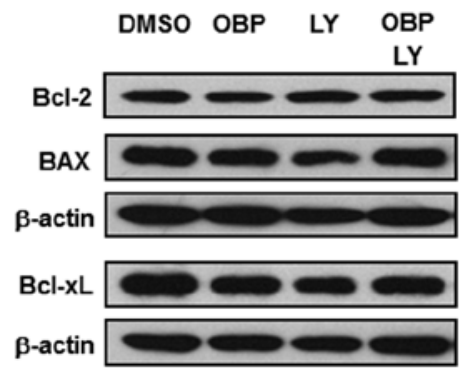

C

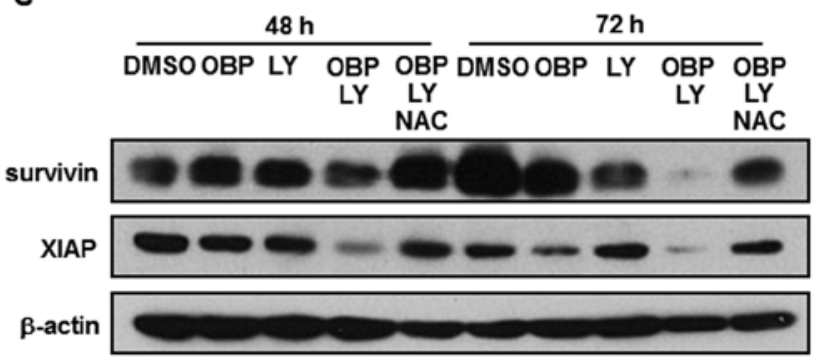

Figure 4. OBP-801 and LY294002 downregulate the expression of survivin and XIAP. (A) Western blotting of IAP family members (survivin and XIAP). $786-O$ cells were treated with $4 \mathrm{nM} \mathrm{OBP}-801$ and/or $5 \mu \mathrm{M} \mathrm{LY} 294002$ for $48 \mathrm{~h}$. $\beta$-actin is shown as a loading control. (B) Western blotting of Bcl-2 family members (Bcl-2, BAX and Bcl-xL). 786-O cells were treated as shown in (A) $\beta$-actin is shown as a loading control. (C) Western blotting of survivin and XIAP. 786-O cells were treated with $4 \mathrm{nM}$ OBP-801 and/or $5 \mu \mathrm{M}$ LY294002 with or without $5 \mathrm{mM} \mathrm{NAC}$ for 48 or $72 \mathrm{~h}$. $\beta$-actin is shown as a loading control.

whereas the co-expression of survivin and XIAP considerably suppressed it. These results suggest that the combination of OBP-801 and LY294002 causes apoptosis at least partially through the downregulation of survivin and XIAP in 786-O cells.

In the combination with LY294002, OBP-801 more strongly induces apoptosis than SAHA in 786-O cells. Suberoylanilide hydroxamic acid (SAHA) is the most clinically used HDAC inhibitor (23). To compare OBP-801 and SAHA in combination with LY294002, we analyzed sub-G1 by flow cytometry. As shown in Fig. 6A, OBP-801 or SAHA alone almost equally induced apoptosis, but co-treatment with OBP-801 and LY294002 more remarkably induced apoptosis than that with SAHA and LY294002 in 786-O cells. These results indicate that OBP-801 is significantly more effective than SAHA in the combination with LY294002 in 786-O cells.
A
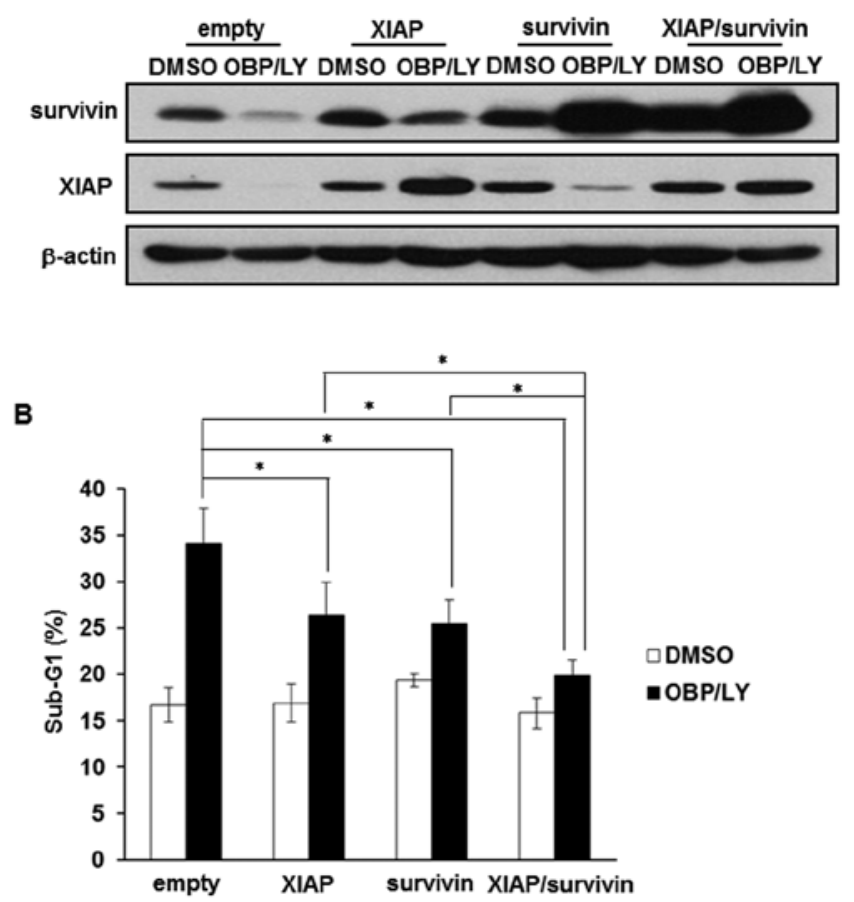

Figure 5. Survivin and XIAP are involved in OBP-801/LY294002-induced apoptosis. (A) 786-O cells were transfected with plasmids as indicated. Twenty-four hours after the transfection, cells were treated with or without $4 \mathrm{nM}$ OBP-801 and $5 \mu \mathrm{M}$ LY294002 for $48 \mathrm{~h}$ and were then harvested, followed by western blotting of survivin and XIAP. $\beta$-actin is shown as a loading control. (B) 786-O cells were transfected as described in (A). Twenty-four hours after the transfection, cells were treated with or without $4 \mathrm{nM}$ OBP-801 and $5 \mu \mathrm{M}$ LY294002 for $48 \mathrm{~h}$. The sub-G1 population was analyzed by flow cytometry. Columns, means of triplicate data; bars, SD; ${ }^{*} \mathrm{P}<0.05$.

\section{Discussion}

HDAC inhibitors have been reported to have potent anticancer activity in various cancer types, but their role as monotherapies appears to be limited. Considering the pleiotropic effects of HDAC inhibitors against malignant tumors, their true therapeutic potential most likely lies in combinations with other anticancer drugs (24). Recent clinical trials have indicated that HDAC inhibitors enhance the antitumor activities of several conventional chemotherapeutic and molecular-target drugs (25-27). Additionally, a previous study showed that HDACs were highly expressed in RCC, suggesting that HDAC inhibitors may be effective on RCC (28).

Recently, the mTOR inhibitors everolimus and temsirolimus have been clinically used as a treatment against RCC, but are not curative. mTOR is known to inhibit the insulin receptor substrate-1 (IRS-1), which plays a key role in transmitting signals from insulin-like growth factor-I (IGF-I) receptors to the PI3K-Akt pathway. Therefore, mTOR inhibitors reactivate the PI3K-Akt pathway resulting in resistance (29). We then selected a PI3K inhibitor as a combination-therapeutic partner of the HDAC inhibitor.

We showed that the OBP-801/LY294002 co-treatment specifically downregulated survivin and XIAP proteins in 786-O cells (Fig. 4A). Furthermore, the overexpression of survivin and/or XIAP reduced the apoptotic response to 
A

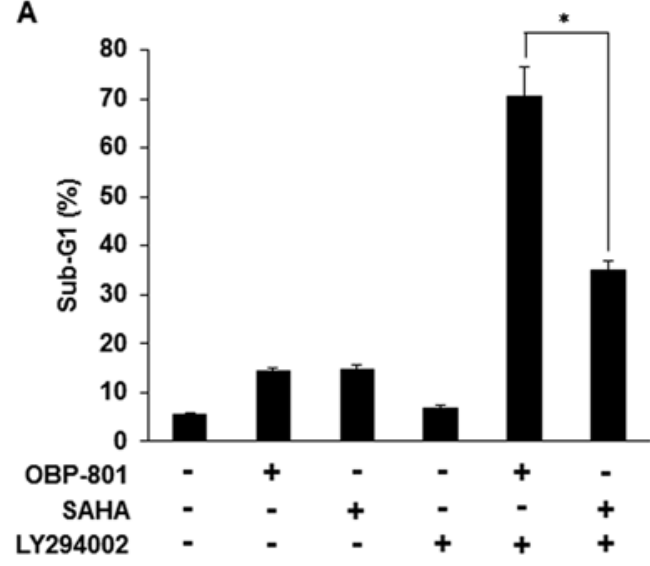

B

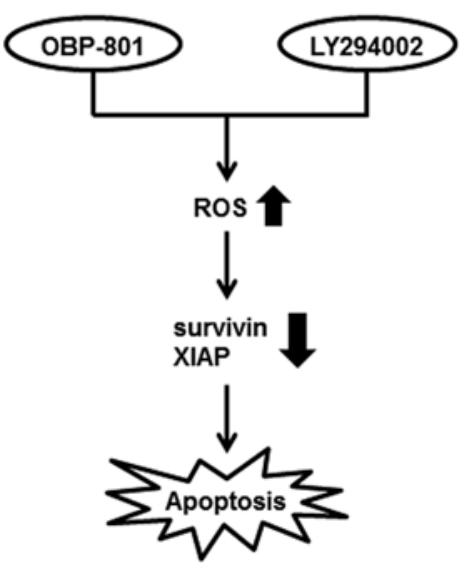

Figure 6. In combination with LY294002, OBP-801 is significantly more effective than SAHA. (A) 786-O cells were treated with $4 \mathrm{nM}$ OBP-801 or $1.6 \mu \mathrm{M}$ SAHA and/or $5 \mu \mathrm{M} \mathrm{LY} 294002$ for $72 \mathrm{~h}$. The sub-G1 population was analyzed by flow cytometry. Columns, means of triplicate data; bars, SD; ${ }^{*} \mathrm{P}<0.05$. (B) The combination of OBP-801 and LY294002 induces intracellular ROS, resulting in a decrease of the expression of survivin and XIAP, finally causing apoptosis in 786-O cells.

OBP-801/LY294002 co-treatment, indicating that the suppression of survivin and XIAP may be attributed to OBP-801/ LY294002-induced apoptosis (Fig. 5B). This is the first report that the downregulation of survivin at least partially contributes to the synergistic effect of an HDAC inhibitor and a PI3K inhibitor. Survivin is a member of the inhibitor of apoptosis (IAP) family and has multiple functions such as the regulation of mitosis and apoptosis $(30,31)$. High expression levels of survivin have been reported to contribute to the resistance of several anticancer agents such as paclitaxel, etoposide and cisplatin (31). Moreover, it has been reported that the overexpression of survivin is associated with disease progression in RCC and may be a prognostic marker in RCC (32). These reports provide the rationale for survivin-targeted therapy in patients with RCC. Therefore, our results show that the combination of OBP-801 with a PI3K inhibitor is promising for the treatment for RCC.

We have additionally found that ROS production contributes to the downregulation of survivin and XIAP by the OBP-801/LY294002 co-treatment (Fig. 4C). It has been also reported that the expression of IAP-family proteins including survivin and XIAP is upregulated by nuclear factor- $\kappa \mathrm{B}$ (NF- $\kappa \mathrm{B})(33-35)$ and that NF- $\kappa \mathrm{B}$ activity is suppressed by ROS (36). Therefore, the downregulation of survivin and XIAP in this study might be caused by the ROS-dependent suppression of NF- $\mathrm{KB}$ activity.

A recent report has shown that the combination of OBP-801 and LY294002 synergistically induces apoptosis through the upregulation of Bim with accumulation of ROS in human endometrial carcinoma HEC-1A cells (37). However, in our experiments, OBP-801/LY294002 co-treatment did not induce Bim expression in RCC 786-O cells (data not shown). These results suggest that there are different mechanisms of apoptosis induced by the OBP-801/LY294002 co-treatment between the two cell lines.

Our results showed that OBP-801 more markedly induced apoptosis than SAHA, the most clinically used HDAC inhibitor, when combined with LY294002 (Fig. 6A). Interestingly, the co-treatment with SAHA and LY294002 did not decrease the expression of survivin and XIAP proteins (data not shown). Therefore, the mechanism for differences in the efficacy of both agents may be attributed to the downregulation of survivin and XIAP.

In conclusion, we demonstrated that the novel HDAC inhibitor OBP-801 and the PI3K inhibitor LY294002 synergistically induced apoptosis by ROS-dependent downregulation of survivin and XIAP in 786-O cells (Fig. 6B). These observations raise the possibility that the combination of OBP-801 and PI3K inhibitors may be promising for the treatment of RCC.

\section{Acknowledgements}

We thank Drs Y. Sowa, S. Yogosawa, M. Tomosugi and M. Koyama for their useful discussion. This study was partly supported by the Japanese Ministry of Education, Culture, Sports, Science and Technology.

\section{References}

1. Rini BI, Campbell SC and Escudier B: Renal cell carcinoma. Lancet 373: 1119-1132, 2009.

2. Gupta K, Miller JD, Li JZ, Russell MW and Charbonneau C: Epidemiologic and socioeconomic burden of metastatic renal cell carcinoma (mRCC): a literature review. Cancer Treat Rev 34: 193-205, 2008

3. Kanno T, Kamba T, Yamasaki T, et al: JunB promotes cell invasion and angiogenesis in VHL-defective renal cell carcinoma. Oncogene 31: 3098-3110, 2012.

4. Mahalingam D, Medina EC, Esquivel JA II, et al: Vorinostat enhances the activity of temsirolimus in renal cell carcinoma through suppression of survivin levels. Clin Cancer Res 16: 141-153, 2010.

5. Carew JS, Esquivel JA II, Espitia CM, et al: ELR510444 inhibits tumor growth and angiogenesis by abrogating HIF activity and disrupting microtubules in renal cell carcinoma. PloS One 7: e31120, 2012.

6. Sosman JA, Puzanov I and Atkins MB: Opportunities and obstacles to combination targeted therapy in renal cell cancer. Clin Cancer Res 13: S764-S769, 2007.

7. Marks PA, Richon VM and Rifkind RA: Histone deacetylase inhibitors: inducers of differentiation or apoptosis of transformed cells. J Natl Cancer Inst 92: 1210-1216, 2000.

8. Koyama M, Izutani Y, Goda AE, et al: Histone deacetylase inhibitors and 15-deoxy-Delta12,14-prostaglandin J2 synergistically induce apoptosis. Clin Cancer Res 16: 2320-2332, 2010.

9. Shindoh N, Mori M, Terada Y, et al: YM753, a novel histone deacetylase inhibitor, exhibits antitumor activity with selective, sustained accumulation of acetylated histones in tumors in the WiDr xenograft model. Int J Oncol 32: 545-555, 2008. 
10. Luo J, Manning BD and Cantley LC: Targeting the PI3K-Akt pathway in human cancer: rationale and promise. Cancer Cell 4: 257-262, 2003.

11. Sourbier C, Lindner V, Lang H, et al: The phosphoinositide 3-kinase/Akt pathway: a new target in human renal cell carcinoma therapy. Cancer Res 66: 5130-5142, 2006.

12. Brognard J, Clark AS, Ni Y and Dennis PA: Akt/protein kinase B is constitutively active in non-small cell lung cancer cells and promotes cellular survival and resistance to chemotherapy and radiation. Cancer Res 61: 3986-3997, 2001.

13. Kulik G, Carson JP, Vomastek T, et al: Tumor necrosis factor alpha induces BID cleavage and bypasses antiapoptotic signals in prostate cancer LNCaP cells. Cancer Res 61: 2713-2719, 2001.

14. Izuishi K, Kato K, Ogura T, Kinoshita T and Esumi $\mathrm{H}$ : Remarkable tolerance of tumor cells to nutrient deprivation: possible new biochemical target for cancer therapy. Cancer Res 60: 6201-6207, 2000.

15. Lee CM, Fuhrman CB, Planelles V, et al: Phosphatidylinositol 3-kinase inhibition by LY294002 radiosensitizes human cervical cancer cell lines. Clin Cancer Res 12: 250-256, 2006.

16. Zhou C, Qiu L, Sun Y, et al: Inhibition of EGFR/PI3K/AKT cell survival pathway promotes TSA's effect on cell death and migration in human ovarian cancer cells. Int J Oncol 29: 269-278, 2006.

17. Wang Q, Li N, Wang X, Kim MM and Evers BM: Augmentation of sodium butyrate-induced apoptosis by phosphatidylinosito 3'-kinase inhibition in the KM20 human colon cancer cell line. Clin Cancer Res 8: 1940-1947, 2002.

18. Park JK, Cho CH, Ramachandran S, et al: Augmentation of sodium butyrate-induced apoptosis by phosphatidylinositol 3-kinase inhibition in the human cervical cancer cell-line. Cancer Res Treat 38: 112-117, 2006.

19. Ozaki K, Kosugi M, Baba N, et al: Blockade of the ERK or PI3K-Akt signaling pathway enhances the cytotoxicity of histone deacetylase inhibitors in tumor cells resistant to gefitinib or imatinib. Biochem Biophys Res Commun 391: 1610-1615, 2010.

20. Wozniak MB, Villuendas R, Bischoff JR, et al: Vorinostat interferes with the signaling transduction pathway of T-cell receptor and synergizes with phosphoinositide-3 kinase inhibitors in cutaneous T-cell lymphoma. Haematologica 95: 613-621, 2010.

21. Rahmani M, Yu C, Reese E, et al: Inhibition of PI-3 kinase sensitizes human leukemic cells to histone deacetylase inhibitormediated apoptosis through p44/42 MAP kinase inactivation and abrogation of p21(CIP1/WAF1) induction rather than AKT inhibition. Oncogene 22: 6231-6242, 2003.

22. Nakata S, Yoshida T, Horinaka M, Shiraishi T, Wakada M and Sakai T: Histone deacetylase inhibitors upregulate death receptor 5/TRAIL-R2 and sensitize apoptosis induced by TRAIL/APO2-L in human malignant tumor cells. Oncogene 23: 6261-6271, 2004.

23. Carew JS, Giles FJ and Nawrocki ST: Histone deacetylase inhibitors: mechanisms of cell death and promise in combination cancer therapy. Cancer Lett 269: 7-17, 2008.
24. Bots M and Johnstone RW: Rational combinations using HDAC inhibitors. Clin Cancer Res 15: 3970-3977, 2009.

25. Ramaswamy B, Fiskus W, Cohen B, et al: Phase I-II study of vorinostat plus paclitaxel and bevacizumab in metastatic breast cancer: evidence for vorinostat-induced tubulin acetylation and Hsp90 inhibition in vivo. Breast Cancer Res Treat 132: 1063-1072, 2012.

26. Rathkopf D, Wong BY, Ross RW, et al: A phase I study of oral panobinostat alone and in combination with docetaxel in patients with castration-resistant prostate cancer. Cancer Chemother Pharmacol 66: 181-189, 2010.

27. Chinnaiyan P, Chowdhary S, Potthast L, et al: Phase I trial of vorinostat combined with bevacizumab and CPT-11 in recurrent glioblastoma. Neurooncology 14: 93-100, 2012.

28. Fritzsche FR, Weichert W, Roske A, et al: Class I histone deacetylases 1,2 and 3 are highly expressed in renal cell cancer. BMC Cancer 8: 381, 2008.

29. Hudes GR: Targeting mTOR in renal cell carcinoma. Cancer 115: 2313-2320, 2009

30. Altieri DC: Survivin, cancer networks and pathway-directed drug discovery. Nat Revi Cancer 8: 61-70, 2008.

31. Mita AC, Mita MM, Nawrocki ST and Giles FJ: Survivin: key regulator of mitosis and apoptosis and novel target for cancer therapeutics. Clin Cancer Res 14: 5000-5005, 2008.

32. Krambeck AE, Dong H, Thompson RH, et al: Survivin and b7-h1 are collaborative predictors of survival and represent potential therapeutic targets for patients with renal cell carcinoma. Clin Cancer Res 13: 1749-1756, 2007.

33. Taniguchi $\mathrm{H}$, Horinaka M, Yoshida $\mathrm{T}$, et al: Targeting the Glyoxalase pathway enhances TRAIL efficacy in cancer cells by downregulating the expression of antiapoptotic molecules. Mol Cancer Ther 11: 2294-2300, 2012.

34. Zhu L, Fukuda S, Cordis G, Das DK and Maulik N: Antiapoptotic protein survivin plays a significant role in tubular morphogenesis of human coronary arteriolar endothelial cells by hypoxic preconditioning. FEBS Lett 508: 369-374, 2001.

35. Stehlik C, de Martin R, Kumabashiri I, Schmid JA, Binder BR and Lipp J: Nuclear factor (NF)-kappaB-regulated X-chromosomelinked iap gene expression protects endothelial cells from tumor necrosis factor alpha-induced apoptosis. J Exp Med 188: 211-216, 1998.

36. Qu Y, Wang J, Ray PS, et al: Thioredoxin-like 2 regulates human cancer cell growth and metastasis via redox homeostasis and NF-kappaB signaling. J Clin Invest 121: 212-225, 2011.

37. Yoshioka T, Yogosawa S, Yamada T, Kitawaki J and Sakai T: Combination of a novel HDAC inhibitor OBP-801/YM753 and a PI3K inhibitor LY294002 synergistically induces apoptosis in human endometrial carcinoma cells due to increase of Bim with accumulation of ROS. Gynecol Oncol 129: 425-432. 2013. 